\title{
Therapeutic Interventions are Important Risk Factors for Delirium in the ICU Setting $\triangle A$ Multicenter Observational Study
}

\section{Ying Liu}

The Affiliated Hospital of Qingdao University

\section{Zhiyong Yuan}

The Affiliated Hospital of Qingdao University

\section{Shixia Cai}

The Affiliated Hospital of Qingdao University

\section{Xiaoning Han}

The Affiliated Hospital of Qingdao University

\section{Kai Song}

Qingdao University

\section{Mei Wang}

Saint Joseph's Healthcare Hamilton

\section{Haifeng Teng}

Weihai Municipal Hospital

\section{Yunbo Sun}

The Affiliated Hospital of Qingdao University

Jinyan Xing ( $\nabla$ xingjy@qdu.edu.cn )

The Affiliated Hospital of Qingdao University

\section{Research}

Keywords: delirium, critical care, intensive care unit, risk factors, prevention

Posted Date: June 11th, 2021

DOl: https://doi.org/10.21203/rs.3.rs-567310/v1

License: (c) (i) This work is licensed under a Creative Commons Attribution 4.0 International License. Read Full License 


\section{Abstract \\ Background}

Delirium is an important independent predictor of negative clinical outcomes in intensive care unit (ICU) patients. The purpose of this study was to investigate the territorial incidence of ICU delirium, its related risk factors, and short-term outcomes in Shandong Province, China, to provide precise information for territorial patient management.

\section{Methods}

A multicenter prospective observational study was conducted. Patients with delirium were defined as any patient with at least one positive CAM-ICU or ICDSC assessment. Demographics, admission clinical data, daily interventions provided to patients and environmental factors were collected.

\section{Results}

From May 1, 2018 to Jan 31, 2020, 536 noncomatose patients were ultimately eligible for the study. One hundred eighteen patients (22\%) experienced delirium at least once. In the univariate analysis, age ( $p=$ $0.009)$, SOFA score $(p=0.006)$, a history of cerebrovascular disease $(p=0.044)$ and impaired renal function ( $p=0.003$ ) were risk factors for delirium. Most therapeutic interventions were linked to delirium in the univariate analysis, including enteral nutrition $(p=0.000)$, artificial airway $(p=0.021)$, nasogastric tube $(p=0.001)$, use of restraint straps $(p=0.000)$, and use of sedative medications, including midazolam $(p=0.003)$, propofol $(p=0.032)$ and butorphanol $(p=0.028)$. Among the patient's vital signs and laboratory examinations performed on the day of assessment, body temperature, BUN levels and CRP levels were risk factors for delirium. Midazolam use, chronic renal insufficiency, physical restraints, nosogastric tube, enteral nutrition, and CRP and BUN levels were factors associated with an increased risk of delirium in the multivariate analysis. The durations of mechanical ventilation and ICU stay in patients with delirium was significantly higher than those in patients without delirium [8 (IQR: 4-14) vs 5 (IQR: $3-10)$ and 9 (IQR: 4-17) vs 6 (IQR: $4-12$ ), $p<0.05]$.

\section{Conclusions}

Delirium was associated with prolonged mechanical ventilation and a prolonged ICU stay. Based on the findings from this study, we should not only reduce the use of sedatives and analgesics but also minimize invasive operations, including the placement of nasogastric tubes, to recover eternal nutrition for ICU patients and avoid physical restraints as much as possible to prevent delirium.

\section{Trial registration:}


This study was registered in the Chinese Clinical Trial Registry (ChiCTR1900021360).

\section{Background}

Delirium has recently been redefined in the DSM-5 as an acute disturbance in attention and awareness, with additional disturbances in cognition not explained by a pre-existing neurocognitive disorder and caused by another medical condition[1]. Delirium impairs the patient's ability to receive, process, store and recall information. Delirium is common among intensive care unit (ICU) patients, depending on the type of ICU, acuity of illness, presence of mechanical ventilation, and other risk factors[2]. The incidence of delirium ranges from $16-80 \%$ in the ICU[2-4]. It can lead to short-term and long-term adverse outcomes in patients. For instance, ICU delirium is associated with nonplanned tube drawing, increased mortality, prolonged hospitalization and mechanical ventilation. It may also increase hospitalization costs and the occurrence of cognitive disorders after discharge from the ICU and even disability after hospitalization[5-8]. Although delirium is associated with a poor outcome, it has not received sufficient attention from clinicians[2, 9]. Delirium is often poorly recognized even by experienced physicians, which may lead to inappropriate treatments and poor outcomes[10].

Over 100 different risk factors have been described for delirium. Barr et al. stated that the risk factors that are positively and significantly associated with the development of delirium in the ICU are (1) pre-existing dementia, (2) a history of hypertension and alcoholism, and (3) a high severity of illness on admission[11]. Acute illness, medications, and environmental effects alter delirium in different ways. Each additional point on the APACHE II score at admission increases the risk of delirium by $5-6 \%$ [12]. A number of investigations have reported a positive relationship between benzodiazepine use and delirium occurrence in critically ill adults[13-14], while Zaal documented that benzodiazepine use, especially with continuous infusion, causes a dose-dependent increase in the risk of delirium [15]. Environmental factors such as daylight and ICU sound may affect the occurrence of delirium. Critically ill patients admitted to single-bed rooms have a lower prevalence of delirium than those admitted to multibed rooms[16].

Numerous pharmacological and nonpharmacological strategies have been investigated as delirium treatments, but consistent results regarding improved outcomes have not been obtained[17]. None of the studied agents significantly improved clinical outcomes, including mortality[18-20]. Therefore, delirium prevention strategies, such as the evaluation and modification of risk factors, are prioritized by current critical care practice guidelines[21].

The risk factors associated with delirium vary depending on the purpose of the study and the selected cohort. The relatively large variation is probably associated with many factors, such as patient populations, screening instruments for delirium (the confusion assessment method for the diagnosis of delirium in the ICU (CAM-ICU) vs Intensive Care Delirium Screening Checklist (ICDSC)), and diagnosis and treatment habits. Risk factors for delirium have gradually attracted the attention of clinicians and researchers in China. According to our previous investigation[22], knowledge, attention, and evaluations of delirium in the ICUs of Shandong Province were significantly deficient, which may explain why 
epidemiological investigations of risk factors for delirium are rare. Thus, the risk factors for delirium in Shandong Province, a northeast seaside province with a population of approximately 100 million people, must be examined to provide unique endemic evidence for the prevention and control of delirium and to explore the incidence and short-term outcomes of patients with ICU delirium in tertiary hospitals.

\section{Methods}

\section{Study design and participants}

Consecutive patients admitted to the participating ICU centers of tertiary hospitals in Shandong Province were evaluated for eligibility from May 1, 2018 to Jan 31, 2020. Before patient recruitment, written informed consent was obtained from each subject or his/her legally authorized representative (surrogate). Adult patients ( $\geq 18$ years old) who were admitted to the ICU for more than 24 hours and signed the informed consent form were enrolled in the study. Patients were excluded from this study if they had 1) coma, dementia, cerebral infarction or hemorrhage, brain trauma, or mental disorders; 2) a previous history of delirium or other mental disorders and long-term use of psychotropic drugs; or 3) were unable to be assessed because of vision, hearing and physical disabilities. All patients were treated according to $A B C D E$ bundles and spontaneous breathing trials (SBTs). The choice of drug dosage and the duration of administration were determined by the physicians.

\section{Delirium screening}

Two of the trained research team members in each participating ICU separately assessed the included patients two times during the day of the investigation. Delirium screening was carried out twice daily at 8:00 AM - 10:00 AM and 14:00-16:00 PM using CAM-ICU and ICDSC. All patients were evaluated for delirium until discharge from the ICU or death. Patients with delirium were defined as any patient with at least one positive CAM-ICU or ICDSC assessment during our trial.

\section{Data collection and quality control}

Data obtained included:1) Baseline characteristics: sex, age, smoking history, obstructive sleep apnea hypopnea syndrome, chronic lung disease, diabetes, heart disease, chronic renal insufficiency, chronic liver disease, cerebrovascular disease, and history of hypertension; 2) Disease condition: principal diagnosis, the indication for ICU hospitalization, trauma, days of ICU stay, APACHE II score, and Sequential Organ Failure Assessment (SOFA) score; 3) Vital signs: temperature and mean arterial pressure; 4) Laboratory tests: $\mathrm{pH}$ value, partial oxygen pressure, oxygen saturation index, and levels of albumin, bilirubin, creatinine, BUN, blood sodium, blood phosphorus, serum cholinesterase, procalcitonin (PCT), C-reactive protein (CRP), lactate, and hemoglobin; 4) Treatment: sedative drugs and dosage, vasoactive drugs and dosage, artificial airway, mechanical ventilation, continuous renal replacement therapy (CRRT), central venous catheter, invasive artery, drainage tube, urinary catheter, stomach catheter, nutrition support therapy, restraint, ability to see the clock, single room, and hormone use; 5) Delirium 
assessment: Richmond Agitation Sedation Scale (RASS), CAM-ICU and ICDSC; and 6) Outcomes (after 28 days): duration of the ICU stay and duration of the hospital stay.

We explored the main risk factors for ICU delirium using all of the related data described above. All data were collected electronically using an electronic clinical report form. Each participating unit had a unique password to log into this online electronic system to ensure confidentiality.

All research team members who executed the assessment of delirium and data collection were experienced professional ICU staff. Each member of the research team was trained on the significance, objectives, specific contents, and methods of the research before and during the study according to the quality control processes executed by the supervisor of the study. Data accuracy and compliance were also guaranteed by the same quality control team and unscheduled meetings of all responsible persons at the participating centers. Every participating site chose a specific date in each month to enroll and assess all patients who were treated in the ICU.

Data on center management were collected and uploaded to each participating site through electronic data online software. Each site chose one day in each month to execute the assessment of delirium in the morning and afternoon, depending on their convenience, but they had to repeat the assessment on the same day in the following months until the end of the study. On the study day, the two researchers from the same center independently began their assessment and data collection at the same time. Every included patient was assessed, and all the related information was recorded at the same time.

\section{Statistics}

Patient characteristics, related covariates and outcomes were summarized using descriptive statistics. $\mathrm{X}^{2}$ tests were used to verify differences between categorical variables that were possibly related to the development of delirium. The t-test was used for normally distributed data, and the Mann-Whitney rank sum test was used for nonnormally distributed data.

Multivariable logistic regression analysis was used to investigate the risk factors associated with the status of delirium, and we used variable selection. Odds ratios are reported with $95 \%$ confidence intervals ( $95 \% \mathrm{Cls}$ ). All tests were 2 -sided, and a $p$ value $<0.05$ indicated statistical significance. Data handling and analyses were performed with SPSS 25.0(IBM SPSS Statistics 25.Ink. Chicago, IL, USA).

\section{Results}

\section{Demographics and Characteristics of Participants}

During the 27-month study period, $11 \mathrm{ICU}$ centers participated the study, including 7 teaching hospitals and 4 territorial medical centers. One thousand one hundred ninety-eight patients were screened and 536 noncomatose patients were ultimately eligible for the study, including 333 male patients $(62.1 \%)$ with a median age of 64 (IQR, 51 to 74) years and an APACHE II score of 13 (IQR, 10 to 19) at the time of admission. The median length of ICU stay was 8 days (IQR, 3 to 17). One hundred eighteen patients 
(22\%) experienced delirium at least once during the ICU stay. The characteristics of all patients are shown in Table 1.

Table 1

Demographics and characteristics of the study participants

\begin{tabular}{|ll|}
\hline Characteristics & All patients \\
\hline Basic & \\
\hline Characteristics & 64 (IQR, 51 to 74$)$ \\
\hline Male sex (percent) & $333(62.1 \%)$ \\
\hline Smoking & $155(28.9 \%)$ \\
\hline Severity of illness at enrollment & \\
\hline APACHE II score & $13($ IQR, 10 to 19$)$ \\
\hline SOFA score & $4($ IQR, 2 to 6$)$ \\
\hline ICU length of stay (days) & $8($ IQR, 3 to 17$)$ \\
\hline Complications & \\
\hline Hypertension & $187(34.9 \%)$ \\
\hline Diabetes & $102(19 \%)$ \\
\hline Snoring & $92(17.2 \%)$ \\
\hline Chronic lung disease & $72(13.4 \%)$ \\
\hline Chronic liver disease & $32(6 \%)$ \\
\hline Impaired renal function & $53(9.9 \%)$ \\
\hline Heart disease & $116(21.6 \%)$ \\
\hline Cerebrovascular disease & $64(11.9 \%)$ \\
\hline Trauma & $125(23.3 \%)$ \\
\hline SalvSis & \\
\hline
\end{tabular}

\section{Univariate Analysis of the Risk Factors for Delirium}

The risk factors for delirium were divided into predisposing factors and precipitating factors.

Predisposing factors were defined as baseline vulnerability factors that existed prior to admission and were difficult to change. Harmful operations or hospitalization-related factors occurring during the course of illness are defined as precipitating factors, including factors related to therapeutic interventions and 
other factors related to critical illness. Precipitating factors are potentially modifiable, allowing clinicians to improve practices and implement preventive measures.

As shown in Table 2, compared with patients without delirium, patients with delirium were older [66 (57$76)$ vs $63(49-73)$ years] and had more serious SOFA score [5 (3-6) vs $4(2-6)]$. They were also more likely to have a history of cerebrovascular disease and impaired renal function. No difference in APECHE II scores was observed between the two groups.

Table 2

Comparison of predisposing factors associated with delirium

\begin{tabular}{|llll|}
\hline Characteristics & Delirium & No Delirium & $\boldsymbol{p}$ \\
\hline Basic & & & \\
Characteristics & & & \\
\hline Age & $66(57-76)$ & $63(49-73)$ & 0.009 \\
\hline Male sex (percent) & $78(66.1 \%)$ & $255(61.0 \%)$ & 0.184 \\
\hline Smoking & $36(23.2 \%)$ & $82(21.5 \%)$ & 0.373 \\
\hline Severity of illness at enrollment & & & \\
\hline APACHE II score & $13(10-19)$ & $13(9-18)$ & 0.379 \\
\hline SOFA score & $5(3-6)$ & $4(2-6)$ & 0.006 \\
\hline Complications & & & \\
\hline Hypertension & $49(41.5 \%)$ & $138(33.0 \%)$ & 0.055 \\
\hline Diabetes & $28(23.7 \%)$ & $74(23.3 \%)$ & 0.092 \\
\hline Snoring & $25(21.2 \%)$ & $67(16.0 \%)$ & 0.121 \\
\hline Chronic lung disease & $16(13.6 \%)$ & $56(13.4 \%)$ & 0.534 \\
\hline Chronic liver disease & $5(4.2 \%)$ & $27(6.5 \%)$ & 0.255 \\
\hline Impaired renal function & $21(17.8 \%)$ & $32(7.6 \%)$ & 0.003 \\
\hline Heart disease & $28(23.7 \%)$ & $88(21.1 \%)$ & 0.306 \\
\hline Cerebrovascular disease & $20(16.9 \%)$ & $44(10.5 \%)$ & 0.044 \\
\hline Trauma & $31(26.3 \%)$ & $94(22.5 \%)$ & 0.230 \\
\hline
\end{tabular}

In addition to these routine, widely studied risk factors, we also evaluated daily interventions.

Differences between the delirium and nondelirium group were observed in most of these interventions, including sedatives and analgesics such as midazolam, propofol and butorphanol; enteral nutrition; 
artificial airway; nasogastric tube, use of restraint straps, and the ability of patients to see the clock. Results are shown in Table 3.

Table 3

Comparison of precipitating factors associated with delirium

\begin{tabular}{|c|c|c|c|}
\hline Characteristics & Delirium & No Delirium & $p$ \\
\hline \multicolumn{4}{|c|}{ Sedative and analgesic drugs } \\
\hline Midazolam & $24(20.3 \%)$ & $42(10.0 \%)$ & 0.003 \\
\hline Dexmedetomidine & $49(41.5 \%)$ & $142(43.0 \%)$ & 0.081 \\
\hline Propofol & $21(17.8 \%)$ & $45(10.8 \%)$ & 0.032 \\
\hline Fentanyl & $11(9.3 \%)$ & $20(4.8 \%)$ & 0.055 \\
\hline Butorphanol & $17(14.4 \%)$ & $33(7.9 \%)$ & 0.028 \\
\hline \multicolumn{4}{|l|}{ Vasoactive Drugs } \\
\hline noradrenaline & $14(11.9 \%)$ & $38(9.1 \%)$ & 0.231 \\
\hline \multicolumn{4}{|l|}{ Daily Interventions } \\
\hline Hormones & $20(16.9 \%)$ & $96(23.0 \%)$ & 0.099 \\
\hline Enteral nutrition & $43(36.4 \%)$ & $254(60.8 \%)$ & 0.000 \\
\hline Artificial airway & $55(46.6 \%)$ & $149(35.6 \%)$ & 0.021 \\
\hline $\begin{array}{l}\text { Mechanical } \\
\text { ventilation }\end{array}$ & $45(38.1 \%)$ & $125(29.9 \%)$ & 0.058 \\
\hline CRRT & $14(11.7 \%)$ & $43(10.3 \%)$ & 0.366 \\
\hline $\begin{array}{l}\text { Deep intravenous } \\
\text { catheter }\end{array}$ & $58(49.2 \%)$ & $170(40.7 \%)$ & 0.062 \\
\hline Invasive artery & $61(51.7 \%)$ & $221(52.9 \%)$ & 0.451 \\
\hline Urinary catheter & $99(83.9 \%)$ & $331(79.2 \%)$ & 0.158 \\
\hline Nasogastric tube & $90(76.3 \%)$ & $254(60.8 \%)$ & 0.001 \\
\hline Drainage tube & $44(37.3 \%)$ & $170(40.7 \%)$ & 0.290 \\
\hline Restraint & 107 (90.7\%) & $240(57.4 \%)$ & 0.000 \\
\hline \multicolumn{4}{|l|}{ ICU Environment } \\
\hline Private room & $54(45.8 \%)$ & $226(54.1 \%)$ & 0.529 \\
\hline Able to see the clock & $40(33.9 \%)$ & 188 (45.0\%) & 0.020 \\
\hline
\end{tabular}


Among the patient's vital signs and laboratory examinations recorded on the day of assessment( shown in Table 4), body temperature and BUN and CRP levels were risk factors for delirium.

Table 4

Comparison of test results on the day of evaluation

\begin{tabular}{|llll|}
\hline Characteristics & Delirium & No Delirium & $p$ \\
\hline Temperature (degrees Celsius) & $37.0(36.7-37.4)$ & $36.9(36.6-37.2)$ & 0.042 \\
\hline $\begin{array}{l}\text { Mean arterial pressure } \\
\text { (mmHg) }\end{array}$ & $85(78-95)$ & $85(77-92)$ & 0.219 \\
\hline lactate (mmol/L) & $1.0(0.8-1.3)$ & $1.0(0.8-1.4)$ & 0.234 \\
\hline Oxygen Saturation Index (specify) & $258.5(216-313)$ & $275(218-371)$ & 0.073 \\
\hline Partial oxygen pressure & $94.7(76.2-110)$ & $93.0(78.0-114)$ & 0.753 \\
(mmHg) & & & \\
\hline Albumin (g/dL) & $29.99(27.00-33.00)$ & $30.60(27.50-33.30)$ & 0.269 \\
\hline Bilirubin (g/dL) & $15.40(9.90-28.8)$ & $14.60(9.20-23.90)$ & 0.473 \\
\hline Creatinine $(\mu \mathrm{mol} / \mathrm{L})$ & $72(54-95)$ & $70(52-93)$ & 0.569 \\
\hline BUN (mg/dL) & $9.60(6.44-17.15)$ & $7.78(5.17-14.00)$ & 0.004 \\
\hline Blood sodium levels (mmol/L) & $139.0(136.0-143.0)$ & $138.6(135.4-142.0)$ & 0.130 \\
\hline Phosphorus levels (mg/dL) & $1.01(0.80-1.23)$ & $0.95(0.73-1.19)$ & 0.195 \\
\hline Serum cholinesterase (IU/L) & $4331(2978-5544)$ & $4535(3313-5859)$ & 0.224 \\
\hline Procalcitonin (PCT) ( $\mu \mathrm{gg} / \mathrm{L})$ & $0.38(0.11-1.11)$ & $0.26(0.10-0.86)$ & 0.115 \\
\hline C-Reactive Protein $(\mathrm{CRP})(\mathrm{mg} / \mathrm{dL})$ & $56.4(26.3-97.0)$ & $36.8(10.5-75.3)$ & 0.001 \\
\hline pH value & $7.45(7.40-7.48)$ & $7.44(7.40-7.47)$ & 0.505 \\
\hline Hemoglobin level (g/dL) & $94(85-111)$ & $96(84-114)$ & 0.661 \\
\hline
\end{tabular}

\section{Multivariate analysis}

Risk factors with $p<0.05$ in the univariate analysis were included in the regression analysis. The multivariate logistic regression analysis revealed 6 factors that were retained in the optimized multivariate model, of which enteral nutrition was a protective factor, while midazolam, chronic renal insufficiency, and restraint were risk factors. Shown in Table 5. 
Table 5

Multivariate Model for Logistic Regression Analysis Predicting Major Factors

\begin{tabular}{|c|c|c|c|c|c|c|c|c|}
\hline \multirow[t]{2}{*}{$\begin{array}{l}\text { Logistic } \\
\text { regression } \\
\text { analysis }\end{array}$} & \multirow{2}{*}{$\begin{array}{l}\text { Coefficient of } \\
\text { the Logistic } \\
\text { Regression } \\
\text { Analysis (B) }\end{array}$} & \multirow[t]{2}{*}{$\begin{array}{l}\text { se } \\
\text { of } B\end{array}$} & \multirow[t]{2}{*}{ Wald } & \multirow[t]{2}{*}{ df } & \multirow[t]{2}{*}{$p$} & \multirow[t]{2}{*}{$\begin{array}{l}\text { Estimated } \\
\text { OR } \\
\text { Exp(B) }\end{array}$} & \multicolumn{2}{|c|}{$\begin{array}{l}95 \% \mathrm{Cl} \text { for } \\
\operatorname{Exp}(\mathrm{B})\end{array}$} \\
\hline & & & & & & & Lower & Upper \\
\hline BUN & -.031 & .010 & 8.936 & 1 & .003 & .970 & .951 & .990 \\
\hline CRP & -.007 & .002 & 8.251 & 1 & .004 & .993 & .989 & .998 \\
\hline Midazolam & .755 & .325 & 5.406 & 1 & .020 & 2.127 & 1.126 & 4.018 \\
\hline Restraint & 1.880 & .356 & 27.868 & 1 & .000 & 6.556 & 3.262 & 13.178 \\
\hline $\begin{array}{l}\text { Chronic Renal } \\
\text { Insufficiency }\end{array}$ & .901 & .360 & 6.279 & 1 & .012 & 2.463 & 1.217 & 4.983 \\
\hline Enteral nutrition & -.859 & .249 & 11.884 & 1 & .001 & .424 & .260 & .690 \\
\hline constant & .522 & .514 & 1.032 & 1 & .310 & 1.686 & & \\
\hline
\end{tabular}

\section{Consequences of delirium}

The duration of mechanical ventilation in patients with delirium was significantly higher than that in patients without delirium [9 (IQR: $4-17$ ) days vs $6(4-12)$ days, $p<0.05]$, and the length of stay in the ICU was longer.Shown in Table6.

Table 6

Comparison of ICU stay and duration of mechanical ventilation

\begin{tabular}{|llll|}
\hline Consequences & Delirium & No Delirium & $\boldsymbol{p}$ \\
\hline duration of ICU stay & $9(4-17)$ & $6(4-12)$ & 0.017 \\
\hline duration of mechanical ventilation & $8(4-14)$ & $5(3-10)$ & 0.035 \\
\hline
\end{tabular}

\section{Discussion}

Shandong Province is one of the most populous provinces in China, with a population of approximately 100 million, and it is the origin of Confucian culture. Delirium is associated with culture because it deeply affects the population at almost every level, especially at the psychological level[23]. Therefore, an investigation of delirium in Shandong Province has special meaning. To the best of our knowledge, this study is the first Chinese multicenter analysis of delirium.

Approximately $22 \%$ of patients experienced delirium at least once during the ICU stay, similar to our previous study (28.75\%)[22]. Patients with delirium were likely to have a longer duration of mechanical ventilation, ICU stay, and hospital stay. In the univariate analysis, age, SOFA score, a history of 
cerebrovascular disease and impaired renal function were risk factors for delirium. Most therapeutic interventions were associated with delirium in the univariate analysis, including enteral nutrition, artificial airways, nasogastric tubes, use of restraint straps and sedative medications, such as midazolam, propofol and butorphanol. Among the patient's vital signs and laboratory examinations reported on the day of assessment, body temperature and BUN and CRP levels were risk factors for delirium.

Consistent with previous studies, age and disease severity have always been risk factors for delirium[24, 25]. Patients with delirium may more frequently be over 65 years of age and have SOFA scores $\geq 2$. Unlike previous studies, no difference in APACHE II scores was observed between patients with and without delirium. We found a large number of errors in the APACHE II scoring process, which may explain this result. We are performing additional research to address this discrepancy.

Although hypertension was identified as a risk factor for ICU delirium in a systematic review[26], hypertension was not associated with delirium in our study, even in the univariate analysis. Moreover, alcohol abuse is one of the most commonly identified risk factors for delirium in Western countries, but in our prior study, we did not find that an alcohol abuse history affected the development of delirium in critically ill Chinese patients, and alcoholics were seldom affected by delirium. Alcohol abuse may be associated with the culture of the society. Therefore, we did not collect any data on alcohol use in the present study.

These risk factors, such as age and severity of illness, are uncontrollable or even immutable for ICU patients. One of the aims of the current study is to differentiate modifiable risk factors and treat these factors in a systemic manner.

Midazolam, butorphanol, and propofol were risk factors for delirium in the univariate analysis. Consistent with other studies, midazolam remained an independent risk factor for delirium in the multivariate analysis[15]. Benzodiazepines and propofol have high affinities for the $y$-aminobutyric acid receptor in the central nervous system, and their administration leads to alterations in the levels of numerous neurotransmitters thought to be deliriogenic[27]. Thus, reducing sedative exposure might be an important intervention to improve patient outcomes. However, in our study, $22.0 \%$ of patients used midazolam, suggesting that we should reduce the use of long-acting sedatives as much as possible in the future and monitor the sedation status of patients more frequently.

Regarding short-acting agents, several studies have shown that dexmedetomidine may be helpful to minimize the depth and duration of sedation with a potential reduction in the time to extubation and days of delirium in the ICU[28, 29]. However, in our study, dexmedetomidine was not correlated with the occurrence of delirium, potentially due to the difference in sedation depth compared with other studies.

Pain is one of the most commonly reported stressors for ICU patients. The negative physiological and psychological consequences of unrelieved pain in ICU patients are significant and long lasting. Therefore, sufficient analgesia should be ensured in all ICU patients, and potentially painful procedures should be mitigated with a preventive analgesic approach[11,30]. We found that the proportion of analgesics used 
in this study was small, and butorphanol was a risk factor in the univariate analysis. Little research has been conducted on butorphanol, especially its effects on delirium. Few studies have compared the superiority of fentanyl to butorphanol in ICU analgesia. Because opioid side effects depend on the dose, these drugs must be compared in equianalgesic doses[31]. We hypothesize that butorphanol may affect delirium by affecting gastrointestinal function. eCASH emphasizes the need to reduce total opioid exposure to avoid adverse effects such as feeding intolerance, constipation and ileus[32].

Gastric tubes, visible clocks, enteral nutrition, physical restraints, artificial airways, and mechanical ventilation were related to delirium in the univariate analysis, consistent with previous research results[22]. Most ICU patients have varying degrees of gastrointestinal dysfunction, and indwelling gastric tubes are common. Few studies have shown the relationship between nutrition and delirium, but our research shows that patients receiving enteral nutrition have a lower risk of delirium than those receiving parenteral nutrition. The specific reasons for providing early EN are to maintain gut integrity and modulate stress and the systemic immune response to attenuate disease severity. A study focusing on the effect of early enhanced enteral nutrition on patients with a head injury found that enhanced EN appears to accelerate neurological recovery and reduce both the incidence of major complications and postinjury inflammatory responses. However, the mechanism is unclear[33].

Therefore, the study described above reminds us that gastrointestinal function, not nutrition modes, may play an important role in delirium. In patients receiving enteral nutrition, the brain-intestinal axis may regulate central nervous system function. However, the relationship between gastrointestinal function and delirium requires further testing.

Patients treated with mechanical ventilation were more likely to develop delirium in the ICU setting than those without mechanical ventilation, consistent with other studies[34]. In the univariate analysis, the incidence of delirium in patients with tracheal intubation or tracheotomy was higher than that in other patients. The probable explanations are that these patients had a more serious illness and needed larger doses of sedatives and analgesics, and the findings may also be related to noise, sleep deprivation, patient-care interaction and the mode of mechanical ventilation. Based on current evidence, the causal relationship between these factors is difficult to distinguish, but we should try different methods, including protocols, to help patients wean from ventilation. Measures should be implemented to shorten the mechanical ventilation time as soon as possible.

The ICU environment will also affect the development of delirium in patients potentially because of different noise and light levels due to the ICU architectural design[16, 35]. Physical restraints are important means to ensure patient safety, handle agitation and prevent unplanned extubation. However, the incidence of delirium is high when physical restraints are used on patients. Restrictions on the use of restraints in the ICU are required to reduce the occurrence of delirium[36].

Even compared with our own previous study, the risk factors that affect delirium are different. The potential explanations are described below. (1) The spectrum of disease varied as the range of participants expanded. (2) Medical staff in different ICUs have different levels of knowledge of delirium 
(as mentioned in our previous study[37]), leading to differences in the implementation of interventions and evaluations, especially delirium evaluations. (3) The occurrence of delirium is related to the territorial culture.

\section{Limitations}

The study also has several limitations. First, as a drawback of observational studies, some unknown or untestable confounding factor(s) affect our internal validity. Second, the study lasted for 27 months, during which changes in therapeutic interventions and cognition might have affected the results. Third, the routine practice for each participating ICU was not the same, which may lead to bias. Additionally, this study was carried out in Shandong Province, China, where the ICU setting may be different from other provinces in China and other countries, which may limit the external validity of the study. Third, we did not follow the patients to determine the long-term effect of delirium on patient prognosis.

Defined delirium risk factors may help practitioners prevent or implement appropriate therapies to reduce delirium in ICU patients and improve their prognosis. We expect that after the success of the study, more ICU medical staff will recognize, pay attention to and routinely evaluate patients for a delirium diagnosis. Therefore, the incidence of delirium will soon be gradually reduced, and the prognosis of ICU patients will be improved.

\section{Conclusions}

Delirium in the ICU setting was associated with a longer duration of mechanical ventilation and a longer ICU stay. In addition to chronic renal insufficiency, CRP and BUN levels, medical interventions, including midazolam usage, physical restraints and the nutrition support mode, were all important risk factors for delirium in our study. Based on the present study, physicians should pay attention not only to reducing the use of sedatives and analgesics but also to minimizing invasive operations, including nasogastric tubes and physical restraints, on patients. This research is limited to Shandong Province, China, and further research on a larger scale might be needed.

\section{Declarations}

\section{Ethics approval and consent to participate}

The study protocol was reviewed and approved by the Qingdao University Research Ethics Board. Before patient recruitment, written informed consent was obtained from each subject or his/her legally authorized representative (surrogate).

\section{Consent for publication}

Not applicable. 


\section{Availability of data and materials}

The datasets used and/or analysed during the current study are available from the corresponding author on reasonable request.

\section{Competing interests}

The authors declare that they have no competing interests.

\section{Funding}

Not applicable.

\section{Authors' contributions}

JYX, HFT and YBS were involved in the study design and protocol development. KS and MW conducted the statistical analysis.YL and CSX wrote the manuscript. ZYY and XNH contributed to the revision and approval of the final manuscript. The author(s) read and approved the final manuscript.

\section{Acknowledgments}

All authors critically assessed and approved the final study design and manuscript. We acknowledge all the staff at the 11 ICU centers that participated in the study.

\section{Authors' information}

${ }^{1}$ Department of Critical Care Medicine, the Affiliated Hospital of Qingdao University, 16 Jiangsu Road, Qingdao, Shandong Province, China. ${ }^{2}$ School of Mathematics and Statistics, Qingdao University, 308 Ningxia Road, Qingdao, Shandong Province, China. ${ }^{3}$ Research Institute, St. Joseph's Healthcare Hamilton, 50 Charlton Avenue East, Hamilton, ON L8N 4A6, Canada. ${ }^{4}$ Department of Health Research Methods, Evidence, and Impact (HEI), McMaster University, 1280 Main Street West, Hamilton, ON L8S 4K1, Canada. ${ }^{5}$ Department of Intensive Care Unit, Weihai Municipal Hospital, 70 HePing Road, Weihai City, Shandong Province, China.

\section{References}

1. American Psychiatric Association. Diagnostic and Statistical Manual of Mental Disorders. 5th ed.: American Psychiatric Association; 2013. 
2. Nicholas JQ,Benjamin Hohlfelder,Matthew R Wanek,Prescribing Practices of Valproic Acid for Agitation and Delirium in the Intensive Care Unit.Ann Pharmacother.2021 Mar;55(3):311-317.

3. Card E, Pandharipande P, Tomes $C$, et al. Emergence from general anaesthesia and evolution of delirium signs in the post-anaesthesia care unit. Br J Anaesth. 2015;115(3):411-7.

4. Salluh JI, Soares M, Teles JM, et al. Delirium Epidemiology in Critical Care Study Group. Delirium. epidemiology in critical. care (DECCA): an international study. Crit Care. 2010;14(6):R210.

5. Girard TD, Jackson JC, Pandharipande PP, et al. Delirium as a predictor of long-term cognitive impairment in survivors of critical illness. Crit Care Med. 2010;38(7):1513-20.

6. Katarzyna Kotfis1. Annachiara Marra1,Eugene Wesley Ely,ICU delirium - a diagnostic and therapeutic challenge in the intensive care unit. Anaesthesiol Intensive Ther. 2018;50(2):160-7.

7. Pisani MA, Murphey TE, Araujo KLB, et al. Benzodiazepine and opoid use and the duration of ICU delirium in an older population. Crit Care Med. 2009b;37:177-83.

8. Zaal IJ, Tekatli H, van der Kooi AW, et al. Classification of daily mental status in critically ill patients for research purposes. J Crit Care. 2015c;30:375-80.

9. Salluh JIF, Wang H, Schneider EB, et al. Outcome of delirium in critically ill patients: systematic review and meta-analysis. BMJ. 2015;350:h2538.

10. Slooter AJC, Van De Leur RR, Zaal IJ. Delirium in critically ill patients. Handb Clin Neurol. 2017;141:449-66.

11. Barr J, Fraser GL, Puntillo K, Ely EW, Gelinas C, Dasta JF, Davidson JE, Devlin JW, Kress JP, Joffe AM, et al. Clinical practice guidelines for the management of pain, agitation, and delirium in adult patients in the intensive care unit. Critical care medicine. 2013;41(1):263-306.

12. Ely EW, Gautam S, Margolin R, et al.The impact of delirium in the intensive care unit on hospital length of stay. Intensive Care Med 27: 1892-1900.

13. Pandharipande $P$, Shintani A, Peterson $J$, et al. Lorazepam is an independent risk factor for transitioning to delirium in intensive care unit patients. Anesthesiology. 2006;104:21-6.

14. Seymour CW, Pandharipande PP, Koestner T, et al. Diurnal sedative changes during intensive care: impact on liberation from mechanical ventilation and delirium. Crit Care Med. 2012;40:2788-96.

15. Zaal IJ, Devlin JW, Hazelbag M, et al. Benzodiazepine-associated delirium in critically ill adults. Intensive Care Med2015 Dec. 2015a;41(12):2130-7.

16. Pedro Caruso,Lilian Guardian,Tatiane Tiengo,ICU architectural design affects the delirium prevalence: a comparison between single-bed and multibed rooms. Crit Care Med.2014 Oct;42(10):2204-10.

17. Roberts B, Rickard CM, Rajbhandari D, Turner G, Clarke J, Hill D, Tauschke C, Chaboyer W, Parsons R. Multicentre study of delirium in ICU patients using a simple screening tool. Australian critical care: official journal of the Confederation of Australian Critical Care Nurses 2005, 18(1):6, 8-9, 11-14 passim.

18. Hayhurst CJ, Pandharipande PP, Hughes CG. Intensive Care Unit Delirium: A Review of Diagnosis, Prevention, and Treatment. Anesthesiology. 2016;125(6):1229-41. 
19. Friedman JI, Soleimani L, McGonigle DP, Egol C, Silverstein JH. Pharmacological treatments of nonsubstance-withdrawal delirium: a systematic review of prospective trials. Am J Psychiatry. 2014;171(2):151-9.

20. Mu JL, Lee A, Joynt GM. Pharmacologic agents for the prevention and treatment of delirium in patients undergoing cardiac surgery: systematic review and meta analysis. Critical care medicine. 2015;43(1):194-204.

21. Zaal IJ, Devlin JW, Peelen LM, Slooter AJ. A systematic review of risk factors for delirium in the ICU. Critical care medicine. 2015;43(1):40-7.

22. Jinyan Xing,Zhiyong Yuan,Yaqi Jie,Risk factors for delirium: are therapeutic interventions part of it? Neuropsychiatric Disease and Treatment 2019:15 1321-1327.

23. Carla Reigada,Paula Sapeta,Carlos Centeno:The value of the ethnographic approach to research issues in palliative care.Curr Opin Support Palliat Care. 2019 Dec;13(4):337-343.

24. Zhang Z, Pan L, Deng H, Ni H, Xu X. Prediction of delirium in critically ill patients with elevated Creactive protein. J Crit Care. 2014;29(1):88-92.

25. Kanova M, Sklienka P, Roman K, Burda M, Janoutova J. Incidence and risk factors for delirium development in ICU patients - a prospective observational study. Biomed Pap Med Fac Univ Palacky Olomouc Czech Repub. 2017;161(2):187-96.

26. Pandharipande $P$, Cotton $B$, Shintani $A$, et al. Prevalence and risk factors for development of delirium in surgical and trauma intensive care unit patients. J Trauma. 2008;65:34-41.

27. Andresen JM, Girard TD, Pandharipande PP, Davidson MA, Ely EW, Watson PL. Burst suppression on processed electroencephalography as a predictor of postcoma delirium in mechanically ventilated ICU patients. Crit Care Med. 2014;42:2244-51.

28. Riker RR, Shehabi Y, Bokesch PM, Ceraso D, Wisemandle W, Koura F, et al. Dexmedetomidine vs midazolam for sedation of critically ill patients: a randomized trial. JAMA. 2009;301:489-99.

29. Pandharipande PP, Pun BT, Herr DL, Maze M, Girard TD, Miller RR, et al. Effect of sedation with dexmedetomidine vs lorazepam on acute brain dysfunction in mechanically ventilated patients: the MENDS randomized controlled trial. JAMA. 2007;298:2644-53. 43.

30. Ralf Baron,Andreas Binder,Rolf Biniek,Evidence and consensus based guideline for the management of delirium, analgesia, and sedation in intensive care medicine. Revision 2015 (DAS-Guideline 2015) short version,Ger Med Sci,2015 Nov 12;13:Doc19.

31. Schnabel A, ReichI SU, Zahn PK,Side Effect Rates of Opioids in Equianalgesic Doses via Intravenous Patient-Controlled Analgesia: A Systematic Review and Network Meta-analysis,Anesth Analg,2019 Oct;129(4):1153-1162.

32. JeanLouis V, Shehabi Y. Timothy S. Walsh,Comfort and patient-centred care without excessive sedation: the eCASH concept,Intensive Care Med (2016) 42:962-971.

33. S J Taylor1,S B Fettes1,C Jewkes1,R J Nelson,Prospective, randomized, controlled trial to determine the effect of early enhanced enteral nutrition on clinical outcome in mechanically ventilated patients suffering head injury,Crit Care Med,1999 Nov;27(11):2525-31. 
34. Ryosuke Tsuruta,Takashi Nakahara, Takashi Miyauchi,Prevalence and associated factors for delirium in critically ill patients at a Japanese intensive care unit.Gen Hosp Psychiatry,Nov-Dec 2010;32(6):607-11.

35. Van Enk RA, Steinberg F. Comparison of private room with multiplebed ward neonatal intensive care unit. HERD. 2011;5:52-63.

36. Yanbin Pan,Zhixia Jiang,Changrong Yuan,Influence of physical restraint on delirium of adult patients in ICU: A nested case-control study,J Clin Nurs,2018 May;27(9-10):1950-1957. doi: 10.1111/jocn.14334. 